\title{
Weekly Administration of Docetaxel and Epirubicin as First-Line Treatment for Hormone-Refractory Prostate Carcinoma
}

\author{
B. Neri,* E. Molinara,* P. Pantaleo,* S. Rangan,* A. Crisci, $\dagger$ A. Della Melina, $\dagger$ \\ A. Raugei, $\dagger$ D. Villari, $\dagger$ and G. Nicita $\dagger$ \\ *Department of Oncology, Center for Experimental and Clinical Oncology, University of Florence, Florence, Italy \\ $\dagger$ Department of Urology, University of Florence, Florence, Italy
}

\begin{abstract}
Androgen-independent prostate carcinoma (AICP) is one of the tumors that continue to respond poorly to chemotherapy. Recently, protocols based on the use of docetaxel have significantly improved survival for patients in this disease. In other types of neoplastic disease, combined therapy with taxanes and anthracycline derivatives has been shown to produce additive effects in terms of growth inhibition, and superior tolerability when associated with weekly administration schedules. These findings prompted us to examine the tolerability and efficacy of weekly treatment of AICP with docetaxel (DOX) plus epirubicin (EPI). We enrolled 35 chemotherapy-naive men with AICP (mean age 72 years, range 68-77) and normal hepatic, renal, and cardiac function. The chemotherapy protocol provided for the IV administration of DOX $\left(30 \mathrm{mg} / \mathrm{m}^{2}\right)$ and EPI $\left(30 \mathrm{mg} / \mathrm{m}^{2}\right)$ on days 1,8 , and 15 every 28 days. Treatment was continued for 6 months or until disease progression and/or unacceptable toxicity was observed. Serum levels of prostate-specific antigen (PSA) were monitored in all patients, and reductions from baseline values of $>50 \%$ were considered indicative of positive responses to treatment. Thirty-four patients were included in the analysis of toxicity, and objective responses to treatment were assessed in the 28 patients with measurable lesions. Nineteen patients $(56 \%)$ experienced PSA reductions of $>50 \%$ that persisted for more than 4 weeks. The response to therapy was classified as complete in 1 of the 28 patients (4\%) with measurable disease (at the lymph node level). Thirteen others $(13 / 28,46 \%)$ had partial responses, in nine $(32 \%)$ the disease remained unchanged, and progression was observed in the remaining five (18\%); overall response rate was $50 \%(\mathrm{CR}+\mathrm{PR})$. Of the 27 patients with pain at the time of enrollment, $16(59 \%)$ experienced pain reduction during treatment. The median time to disease progression was 11.7 months (95\% CI: 7.7-15.7) while the median survival time was 18.7 months (95\% CI: 12.3-25.1). During the study, four patients developed grade 3 anemia and leukopenia, which was reversible in all cases. Lower grades of asthenia, nausea, vomiting, diarrhea, and peripheral edema were also observed. There were no cases of cardiotoxic effects. Alopecia was frequent but reversible in all cases. The results of this preliminary study indicate that the combined administration of DOX and EPI for treatment of AIPC is effective and well tolerated. The weekly administration of the drug combination appears to be a promising approach to the treatment of these tumors.
\end{abstract}

Key words: Prostate carcinoma; Chemotherapy; Docetaxel; Epirubicin; Weekly regimen

\section{INTRODUCTION}

Carcinoma of the prostate is the major cause of morbidity and mortality among elderly men in Western countries (1), and it has increased by $3 \%$ per year over the past decade (2). This is a hormone-dependent tumor, and androgen blockade is currently the first and the most effective treatment. Although most patients respond positively to this type of treatment, the duration of the response is hard to predict. Data from randomized trials and studies of patients with advanced disease indicate that the median time to progression ranges from 12 to 18 months, and median survival varies from 24 to 36 months (3-5). These data are consistent with the clonal selection model proposed by Isaac and Coffey (6), in which disease progression mainly involves cell populations that are refractory to hormone therapy, a characteristic that is often associated with increased expression of the oncogene BCL-2. Overexpression of this oncogene protects the tumor cells from the apoptotic effects of most chemotherapy agents $(7,8)$. This probably explains why chemotherapy for androgen-independent prostate cancer (AIPC) has thus far produced only transient responses and/or marginal benefits in terms of symptom palliation $(9,10)$.

Recently, however, interest in cytotoxic chemotherapy for prostate cancer has been renewed by the results obtained in clinical trials with the combined use of estra- 
mustine and docetaxel (11-14). These observations prompted us to consider three aspects of the problem. First, the most promising chemotherapeutic agents available today for treatment of AIPC are those that act on the microtubules, docetaxel (DOX) in particular. Second, both DOX and anthracycline derivatives like epirubicin (EPI) can provoke apoptosis in prostate carcinoma cells that overexpress the BCL-2 oncogene $(15,16)$. Third, experimental and clinical studies have shown that, in other types of neoplastic disease, the use of taxanes with anthracycline derivatives produces additive effects against the neoplastic cell clone $(17,18)$. These considerations and the fact the AIPC patients are generally quite elderly led us to evaluate both the tolerability and clinical efficacy of a schedule with DOX plus EPI administered on a weekly basis, which allowed for better control of the comorbidity caused by the toxic effects of the drugs being used.

\section{PATIENTS AND METHODS}

\section{Patients}

Eligible patients had histologically confirmed adenocarcinoma of the prostate with advanced stage and/or metastatic disease that had showed signs of progression during standard hormonal therapy. Additional inclusion criteria were: $<80$ years old; informed consent; Eastern Cooperative Oncology Group (ECOG) score $\leq 2$; absence of brain metastases; normal cardiac, hepatic, renal, and bone marrow function; nNo prior chemotherapy with cytotoxic drugs.

Disease progression was defined by the presence of at least one of the following: an increase of $25 \%$ or more in serum levels of PSA in two consecutive measurements obtained at least 1 week apart; aAn increase of $25 \%$ or more in the size of metastatic lesions or the appearance of new lesions; appearance of new bone lesions documented by bone scan.

Androgen blockade achieved with LH-RH analogs was maintained in all patients.At the time of enrollment and prior to the initiation of treatment, each patient had a thorough physical examination, and standard blood chemistry parameters were measured along with serum levels of testosterone and PSA. The patient also had a total-body bone scan, radiologic studies aimed at locating the cause of pain, and, when indicated, computed tomographic scans of the chest and abdomen.

\section{Treatment Regimen}

DOX $\left(30 \mathrm{mg} / \mathrm{m}^{2}\right)$ and EPI $\left(30 \mathrm{mg} / \mathrm{m}^{2}\right)$ were administered on days 1,8 , and 15 every 28 days. The treatment was repeated for 6 months or until evidence of disease progression, patient refusal, or unacceptable adverse reactions.
DOX was diluted in $500 \mathrm{cc}$ of a saline solution and administered by IV infusion over $2 \mathrm{~h}$, followed by an IV bolus of EPI diluted in $100 \mathrm{cc}$ of saline solution. Premedication for DOX included dexamethasone $(8 \mathrm{mg})$ administered by intramuscular injection the evening before the treatment day and again at the time of treatment. Ondansetron ( $8 \mathrm{mg}$ ) was administered IV at the beginning of each cycle to control nausea and vomiting.

In the presence of grade 3 (or higher) hematological toxicity, the doses of DOX and EPI were reduced by $25 \%$ or treatment was postponed until complete recovery had occurred.

\section{Criteria Used to Assess Toxicity and Response to Treatment}

Vital signs, adverse effects, performance-status scores, and complete blood counts were recorded weekly for each patient. Clinical evaluations, including measurement of PSA and instrumental studies of target lesions, were performed every 4 weeks. Pain responses were evaluated with the orally administered McGill-Melzac questionnaire (19), in which pain is rated according to a 5 -point scale $(0=$ no pain, $1=$ mild, $2=$ discomforting, $3=$ distressing, $4=$ horrible, $5=$ excruciating).

The criteria used in assessing the efficacy of treatment were: reduction of PSA levels by at least $50 \%$ that persisted for at least 4 weeks; pain reduction of two points or more on the McGill Melzack questionnaire.

We also assessed the effect of treatment on measurable lesions in accordance with the WHO criteria for evaluation of the response to treatment of solid tumors.

\section{Statistical Methods}

The study was a nonrandomized, phase II study. The primary end point included the evaluation of the regimen-related toxicities and objective responses, and after time to disease progression (TTP) and overall survival (OS). The sample size was calculated on the assumption that a percentage of objective response variables between $20 \%$ and $40 \%$ could be detected. According to the optional Simon two-step design, if a minimum objective response rate $>20 \%$ was observed in the first 15 patients, an additional 15 patients should be enrolled, and if $>12$ responses were observed in 30 patients $(40 \%)$, the regimen was considered active to be submitted for further evaluation (20). Descriptive statistics was reported as proportions and medians. TTP was defined as the interval between initial treatment and the time of disease progression or death. Survival time was calculated from the date of treatment initiation until the date of the last follow-up evaluation or death. TTP and OS were analyzed according to the Kaplan-Meier method (21). The confidence intervals (CIs) for response rates, TTP, and OS 
were calculated using methods for exact binominal confidence interval (22). Survivors were censored on the date they were last known to be alive.

\section{RESULTS}

The characteristics of the 35 patients enrolled in the trial are reported in Table 1. One patient withdrew from the study after the second week without completing the first cycle of treatment. Of the 34 patients who completed the treatment as planned, 28 had measurable lesions. One patient achieved a complete response (CR rate $=4 \%$ ), and 13/28 had partial responses (PR rate $=$ $46 \%$ ). In nine patients, the disease remained stable (SD rate $=32 \%$ ), and disease progression was observed in the other five ( $\mathrm{PD}=18 \%$ ). Figures 1 and 2 report the curve of median time to progression, which was 11.7 months (range 6.1-20.1, 95\% CI 7.7-15.7), and the median survival time, which was 18.7 months (range 8.1-36+, 95\% CI 12.3-25.1).

Reductions of $>50 \%$ in serum PSA levels were observed in 19/34 patients (56\%), and the mean duration of this response was 5.4 months (range $2.4-7.8,95 \%$ CI $3.6-7.2$ ), and reductions of $<50 \%$ were observed in nine (26\%) others. On the whole, performance status im-

Table 1. Clinical Characteristics of the Patients

\begin{tabular}{lrc}
\hline & $\begin{array}{c}\text { No. } \\
\text { Patients } \\
(\%)\end{array}$ \\
Characteristics & 35 \\
\hline Age (years; median 72, range 68-77) & & \\
ECOG performance status & 11 & $(32)$ \\
0 & 19 & $(54)$ \\
1 & 5 & $(14)$ \\
2 & & \\
Main site of metastases & 20 & $(57)$ \\
Bone & 8 & $(23)$ \\
Soft tissue and/or lymph nodes & 4 & $(12)$ \\
Lung & 3 & $(8)$ \\
Liver & & \\
Hormone therapy (LHRH agonists + & $35(100)$ \\
$\quad$ androgen blockers) & & \\
Baseline serum PSA levels (ng/ml) & 172 \\
Median & $7-1700$ \\
Range & & \\
Baseline pain intensity scores & 8 & $(23)$ \\
Grade 0 & 4 & $(12)$ \\
Grade 1 & 12 & $(33)$ \\
Grate 2 & 8 & $(23)$ \\
Grate 3 & 2 & $(6)$ \\
Grade 4 & 1 & $(3)$ \\
Grade 5 & & \\
\hline
\end{tabular}

*Hormone treatment begun prior to study enrollment was continued during the study. proved during treatment in 16/34 patients (47\%). Specifically, pain improved in 16 of the $27(59 \%)$, remained stable in $15 \%$, and worsened in $26 \%$ (Table 2).

A total of 184 cycles of treatment were administered during the trial, with a mean of 5.4 cycles per patient (3-8 cycles). In three cases (9\%), a $25 \%$ dose reduction was necessary because the patient developed grade 3 hematological toxicity; in two others $(6 \%)$ the treatment was simply postponed for 7 days. Two patients developed grade 3 anemia, and three others experienced nonfebrile leukopenia. Table 3 shows the adverse effects observed during the trial.

\section{DISCUSSION}

Androgen-independent prostate cancer (AIPC) is one of the unresolved problems in the field of treatment for solid tumors. Most chemotherapeutic agents have displayed only marginal activity against these tumors, but in recent years promising results have emerged from studies of taxanes and other chemotherapy agents that act on the microtubules. These agents are the first drugs that seem to be able to increase survival in patients with AIPC $(23,24)$. Anthracycline derivatives have also displayed some degree of efficacy in the treatment of advanced prostate cancer (25), and clinical trials have revealed additive effects when these drugs are used with taxanes in the treatment of other types of tumors $(26,27)$. Therefore, in the present study we evaluated the efficacy and safety of weekly administration of DOX + EPI for treatment of advanced AIPC and control of the pain that often accompanies this disease.

The DOX + EPI regimen was associated with an overall response rate of $50 \%$ ( $1 \mathrm{CR}+13 \mathrm{PR})$. In nine patients, the disease remained stable, and progression was observed in the remaining five (18\%). A reduction of $>50 \%$ in serum PSA levels occurred in 19/34 patients (57\%). Moreover, weekly administration of the two drugs was well tolerated. Ninety-one percent of the patients completed the six cycles of treatment provided for in the study protocol, and none experienced adverse events serious enough to warrant discontinuation of treatment. The most severe adverse events were grade 3 anemia and leukopenia, which occurred in four patients (12\%). All of these episodes resolved completely without hospitalization. There were no cases of thromboembolic complications, signs of cardiotoxicity, or asthenia $>$ grade 2 . This rate is higher than those reported in patients treated with other chemotherapy drugs, especially estramustine (9,11-14), which is also associated with poorly tolerated gastrointestinal side effects.

Of the 28 patients with measurable lesions, one (4\%) had a complete response to treatment, and 13 others (46\%) had partial responses. The overall objective re- 


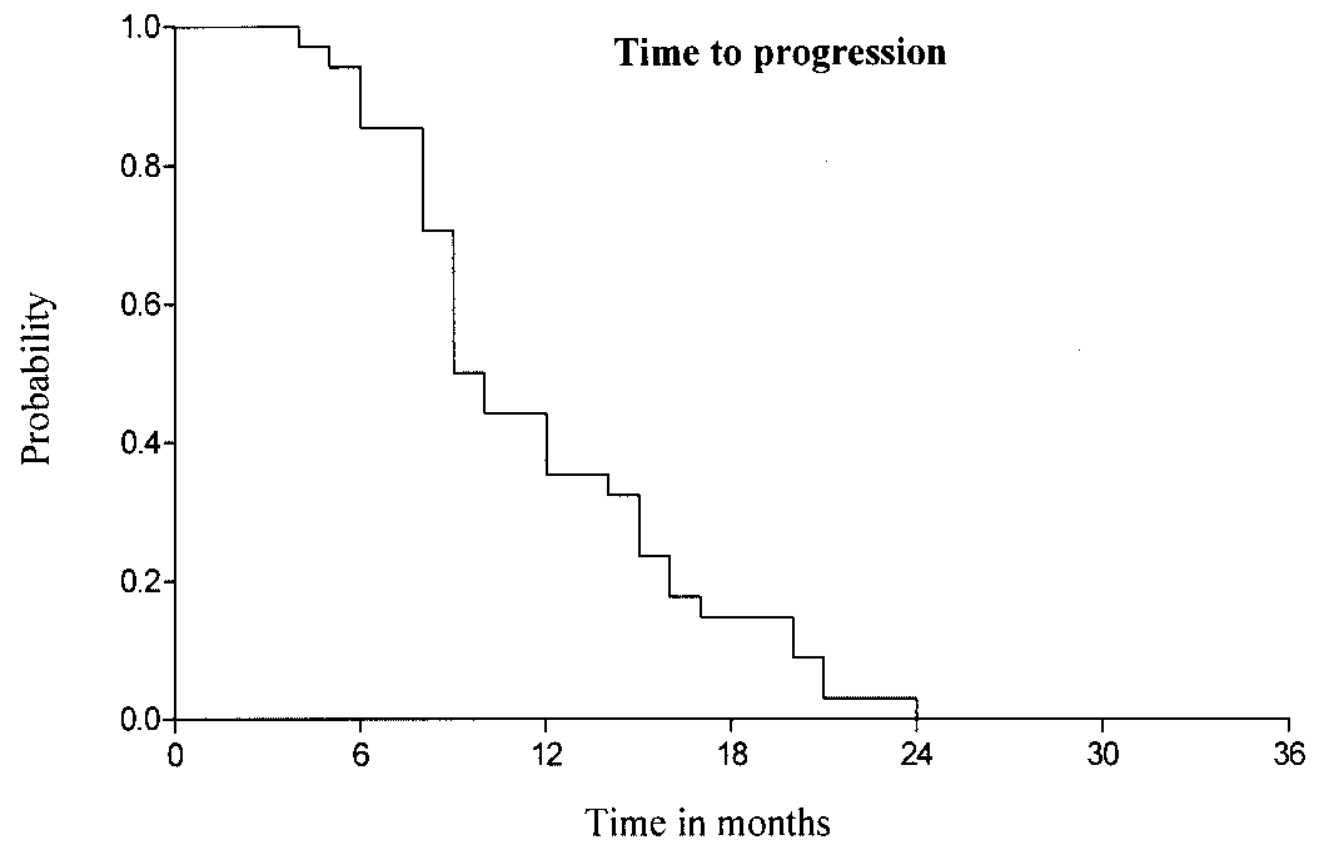

Figure 1. Kaplan-Meier estimates of time to progression among patients with prostate cancer treated with the docetaxel and epirubicin weekly regimen.

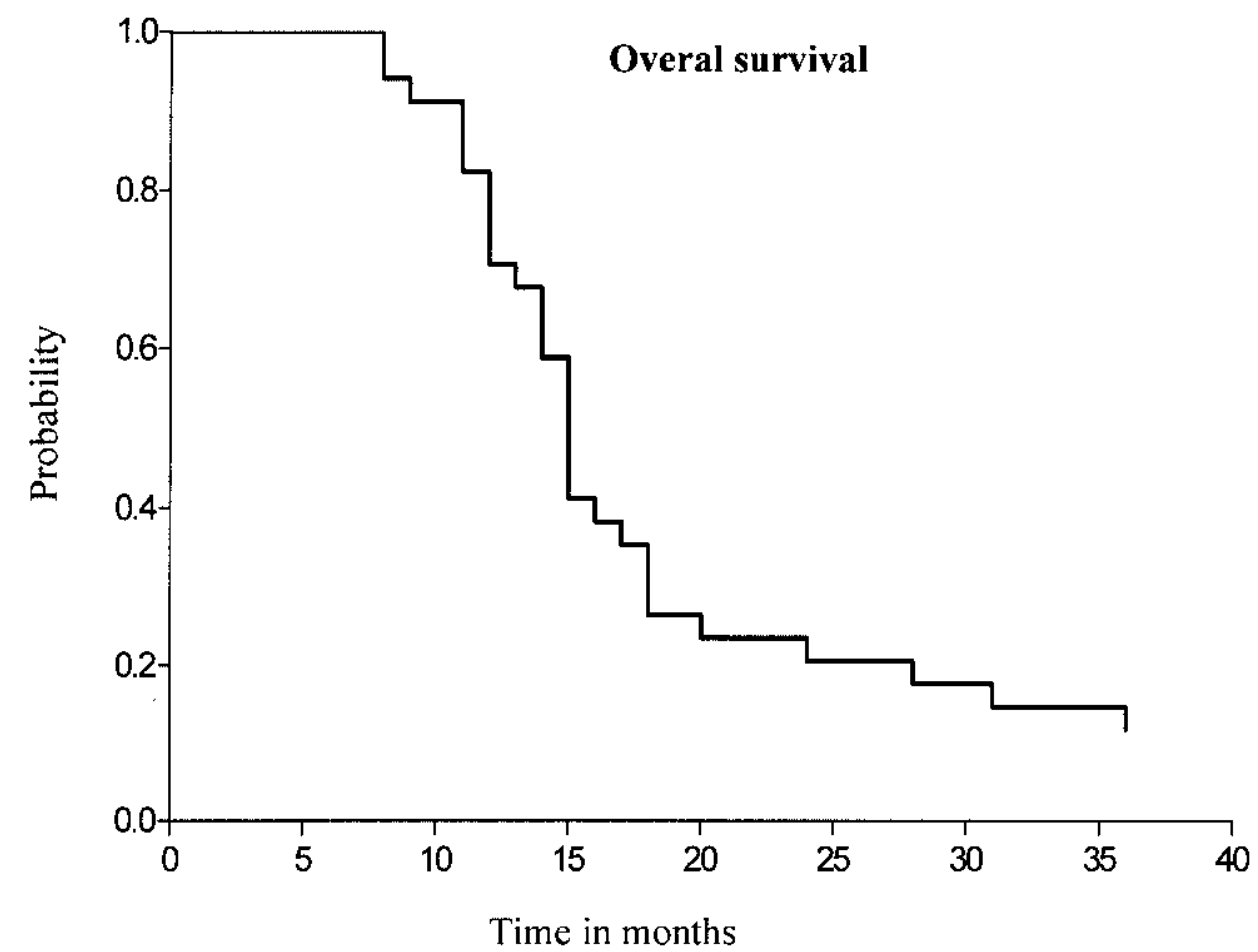

Figure 2. Kaplan-Meier estimates of overall survival among patients with prostate cancer treated with the docetaxel and epirubicin weekly regimen. 
Table 2. Results of Patients Who Could Be Evaluated

\begin{tabular}{|c|c|}
\hline Results & No. $(\%)$ \\
\hline Biological response & $34(100)$ \\
\hline Reduction in serum PSA $>50 \%$ & $19 \quad(56)$ \\
\hline Reduction in serum PSA $<50 \%$ & 9 (26) \\
\hline Response to treatment & $28(100)$ \\
\hline Complete response & $1 \quad(4)$ \\
\hline Partial response & 13 (46) \\
\hline Disease stabilization & $9(32)$ \\
\hline Disease progression & 5 (18) \\
\hline Pain $\dagger$ & $27(100)$ \\
\hline Improved & $16(59)$ \\
\hline Unchanged & 4 (15) \\
\hline Worsened & 7 (26) \\
\hline
\end{tabular}

*Patients with measurable lesions: $28 / 34$.

$\dagger$ Patients with pain at enrollment: 27/34.

sponse rate was thus $50 \%$ ( $1 \mathrm{CR}+13 \mathrm{PR})$. In nine patients, the disease remained stable, and progression was observed in the remaining five (18\%). These results are in line with those achieved with other combined drug protocols based on the use of vinblastine, etoposide, paclitaxel, and docetaxel, with or without estramustine, but most of these approaches are associated with more severe gastrointestinal toxicity and thromboembolic events $(23,24)$. All of the patients with objective responses also experienced decreases in serum PSA levels and, none showed any signs of disease progression during the period of decreased PSA levels. This observation seems to confirm the hypothesis advanced by other investigators that significant reductions in the level of this antigen are associated with better control of symptoms and longer survival.

The results of our study indicate that weekly administration of DOX + EPI can inhibit the progression of

Table 3. Overall Rates of Toxicity

\begin{tabular}{lcccc}
\hline & \multicolumn{4}{c}{$\begin{array}{c}\text { No. (\%) of Patients } \\
\text { With WHO Grade }\end{array}$} \\
\cline { 2 - 5 } Toxicity & 1 & 2 & 3 & 4 \\
\hline Anemia & $15(44)$ & $10(29)$ & $2(6)$ & - \\
Thrombocytopenia & $19(55)$ & $7(20)$ & - & - \\
Leukopenia & $17(50)$ & $14(41)$ & $3(9)$ & - \\
Nausea/vomiting & $13(38)$ & $11(32)$ & - & - \\
Anorexia/weight loss & $21(61)$ & $9(30)$ & - & - \\
Asthenia & $18(52)$ & $13(38)$ & - & - \\
Alopecia & $21(61)$ & $11(32)$ & - & - \\
Cardiac & $15(44)$ & $5(14)$ & - & - \\
Renal & $27(80)$ & - & - & - \\
Neurologic & $19(55)$ & $7(20)$ & - & - \\
\hline
\end{tabular}

AIPC and provide rapid and effective control of pain with provoking uncontrollable or irreversible toxicity. These preliminary findings confirm the results of other studies, which showed that DOX therapy for AIPC is well tolerated and associated with good objective response rates $(25,26)$. Most AIPC patients are elderly men with multiple concomitant diseases, and their bone marrow responses are often reduced. For this reason, we feel that the control of symptoms and good tolerability should be the primary objectives of treatment in AIPC patients. In this context, better results might be achieved by subjecting responding patients to intermittent treatment, as soon as disease progression occurs. The main limitation of our study was the number of enrolled patients. Because the number of patients was so small, no conclusive answer can be drawn. Our future efforts will focus on randomized trials evaluating this DOX + EPI regimen with DOX alone or DOX in other combinations.

ACKNOWLEDGMENTS: The authors acknowledge the assistance of Prof. Grazia Cini for editing the manuscript. Funding for this study was provided by the "Associazione Toscana Ricerche e Cure Oncologiche," Florence, Italy.

\section{REFERENCES}

1. Hanks, G. E.; Myers, C. E.; Scardino, P. T.; et al. Cancer of the prostate. In: De Vita, V. T.; Helman, S.; Rosemberg, S. A., eds. Cancer principles and practice in oncology. Philadelphia: J. P. Lippincott; 1997:1322-1386.

2. Yancik, R. Epidemiology of cancer in the elderly. Current status and projections for the future. RAYS 22(Suppl. 1): 3-9; 1997.

3. Crawford, E. D.; Eisemberger, M. A.; McLeod, D. C.; et al. A controlled randomized trial of leuprolide with and without flutamide in prostatic cancer. N. Engl. J. Med. 321:419-424; 1989.

4. Denis, L.; Whetan, P.; Carniero, D.; et al. Goserelin acetate and flutamide versus bilateral orchiectomy: A Phase III EORTC study (30853). Urology 42:119-130; 1993.

5. McLeod, D. C. Hormonal therapy in the treatment of carcinoma of the prostate. Cancer 75:914-919; 1995.

6. Isaacs, J. T.; Coffey, D. S. Adaptation versus selection as the mechanism responsible for the relapse of prostate cancer to androgen ablation as studied in the Dunning R3327H adenocarcinoma. Cancer Res. 41:5070-5075; 19981.

7. McDonnell, T. J.; Troncoso, P.; Brisbay, S. M.; et al. Expression of proto-oncogene bcl-2in the prostate and its association with emergence of androgen independent prostate cancer. Cancer Res. 52:6940-6944; 1992

8. Colombel, M.; Symmans, F.; Gil, S.; et al. Detection of the apoptosis-suppressing oncoprotein bcl-2 in hormone refractory human prostate cancer. Am. J. Pathol. 143:390400; 1993.

9. Tannok, I. F.; Osoba, D.; Stokler, M. R.; et al. Chemotherapy with mitoxantrone plus prednisone or prednisone alone for symptomatic hormone-resistant prostate cancer: A Canadian randomized trial with palliative end points. J. Clin. Oncol. 14:1756-1764; 1996.

10. Kantoff, P. W.; Halabi, S.; Conoway, M.; et al. Hydrocor- 
tisone with or without mitoxantrone in men with hormone-refractory prostate cancer: Results of the cancer and leukemia group B 9182. J. Clin. Oncol. 17:2506-2513; 1999.

11. Sinibaldi, V. J.; Carducci, M. A.; Moore-Cooper, S.; et al. Phase II evaluation of docetaxel plus one-day oral estramustine phosphate in the treatment of patients with androgen independent prostate carcinoma. Cancer 94:1457$1465 ; 2002$.

12. Tralongo, P.; Bollina, R.; Aiello, R.; et al. Vinorelbine and prednisone in older cancer patients with hormone-refractory metastatic prostate cancer a phase II study. Tumori 89:26-30; 2003.

13. Beer, T. M.; Eilers, K. M.; Garzotto, M.; et al. Weekly high-dose calcitriol and docetaxel in metastatic androgenindependent prostate cancer. J. Clin. Oncol. 21:123-128; 2003.

14. Smith, D. C.; Chay, C. H.; Dunn, R.; et al. Phase II trial of paclitaxel, estramustine, etoposide, and carboplatoin in the treatment of patients with hormone-refractory prostate carcinoma. Cancer 98:269-276; 2003.

15. Tu, S. M.; McConnell, K.; Marin, M. C.; et al. Combination adriamycin and suramin induces apoptosis in bcl-2 expressing prostate carcinoma cells. Cancer Lett. 93:147$155 ; 1995$.

16. Kraus, L. A.; Samuel, S. K.; Schmid, S. M.; et al. The mechanism of action of docetaxel (Taxotere) in xenograft models is not limited to BCL-2 phosphorylation. Invest. New Drugs 21:259-268; 2003.

17. Sackett, D.; Fojo, T. Taxanes. In: Pinedo, H. M.; Longo, D. L.,; Chabner, B. A., eds. Cancer chemotherapy and biological response modifiers. Amsterdam: Elsevier; 1997: 59-79.
18. Conte, P. F.; Michelotti, A.; Baldini, E.; et al. Activity and safety of epirubicin plus paclitaxel in advanced breast cancer. Semin. Oncol. 23:28-32; 1996.

19. Melzack, R. The McGill pain questionnarie major properties and scoring methods. Pain 1:277-299; 1975.

20. Simon, R. Optimal two-stage designs for phase II clinical trials. Control Clin. Trials 10:1-10; 1989.

21. Kaplan, E.; Meier, P. Non-parametric estimation from incomplete observations. J. Am. Stat. Assoc. 53:457-481; 1958.

22. Lenter, C. Exact confidence limits. In: Geigy scientific tables. Switzerland: Ciba-Geigy; 1982:89-102.

23. Tannok, I. F.; de Wit, R.; Berry, W. R.; Horti, J.; et al. Docetaxel plus prednisone or mithoxantrone plus prednisone for advanced prostate cancer. N. Engl. J. Med. 351: 1502-1512; 2004.

24. Petrylak, D. P.; Tangen, C. M.; Hussain, M. H.; et al. Docetaxel and estramustine compared with mithoxantrone and prednisone for advanced refractory prostate cancer. $\mathrm{N}$. Engl. J. Med. 351:1513-1520; 2004.

25. Neri, B.; Barbagli, G.; Bellesi, P.; et al. Weekly epidoxorubicin therapy in hormone-refractory metastatic prostate cancer. Anticancer Res. 17:3817-3820; 1997.

26. Ceresoli, G. L.; Dell'Orso, S.; Passoni, P.; et al. Phase II study of paclitaxel and epirubicin as first-line treatment in patients with metastatic nonsmall cell lung carcinoma. Cancer 89:89-96; 2000.

27. Friedichs, K.; Hozel, F.; Janike, F. Combination of taxanes and anthracyclines in first-line chemotherapy of metastatic breast cancer. Eur. J. Cancer 38:1730-1738; 2003. 